\title{
The Effects of Altitude-related Hypoxia Exposure on the Multiscale Dynamics of Blood Pressure Fluctuation During Sleep: The Observation from a Pilot Study
}

\author{
Qian $\mathrm{Li}^{1}{ }^{1} *$ \\ Zhenxiang Guol'* \\ Fuzheng Liu ${ }^{2}$ \\ Ye $\mathrm{Liu}^{3}$ \\ Dapeng $\mathrm{Bao}^{4}$ \\ Junhong Zhou ${ }^{5}$
}

'Sports Coaching College, Beijing Sport University, Beijing, People's Republic of China; ${ }^{2}$ School of Medical Humanities, Capital Medical University, Beijing, People's Republic of China; ${ }^{3}$ National Team Performance Training Center, National Sports Training Center, General Administration of Sport of China, Beijing, People's Republic of China; ${ }^{4}$ China Institute of Sport and Health Science, Beijing Sport University, Beijing, People's Republic of China; ${ }^{5}$ Hebrew SeniorLife Hinda and Arthur Marcus Institute for Aging Research, Harvard Medical School, Boston, MA, 02I3I, USA

*These authors contributed equally to this work
Correspondence: Dapeng Bao

China Institute of Sport and Health Science, Beijing Sport University, No. 48 Xinxi Road, Haidian District, Beijing, 100084, People's Republic of China

Email baodp@bsu.edu.cn
Purpose: The purpose exposure to hypoxia in high altitudes severely impairs the sleep quality and the related cardiovascular regulation, including the blood pressure (BP) regulation. BP regulation depends upon the continuous interaction of components over multiple temporal scales. As such, the dynamics of BP fluctuation are complex, and BP complexity has been linked to several pathological events. However, the effects of the exposure to hypoxia on BP complexity during sleep remain unknown.

Methods: Twenty-five younger men naïve to high-altitude sleep (apnea severity as assessed by hypoxia apnea index (AHI): normal $=8$; moderate $=9$; severe $=8$ ) completed one nocturnal sleep under each of the three altitudes: 0 (ie, baseline), 2000, and $4000 \mathrm{~m}$. The sleep characteristics and oxygen saturation (ie, $\mathrm{SpO}_{2}$ ) were assessed using polysomnography (PSG). The beat-to-beat BP fluctuation was recorded using a finger-blood-pressure sensor. Multiscale entropy (MSE) was used to characterize the complexity of systolic (SBP) and diastolic (DBP) BP fluctuations, and lower MSE reflected lower complexity.

Results: Compared to 0 -m condition, SBP $(p=0.0003)$ and DBP $(\mathrm{F}=12.1, p=0.0002)$ complexity, $\mathrm{SpO}_{2}(p<0.0001)$ and REM ratio $(p<0.0090)$ were decreased, AHI was increased ( $p=0.0004$ ) in $2000-\mathrm{m}$ and even more in $4000-\mathrm{m}$ conditions. In addition, lower BP complexity was associated with greater AHI $(\mathrm{r}=-0.66 \sim 0.52, p=0.0010)$, lower $\mathrm{SpO}_{2}(\mathrm{r}=0.48 \sim 0.51$, $p=0.0100 \sim 0.0200)$ and lower REM ratio $(\mathrm{r}=0.48 \sim 0.52, p=0.0200)$. Participants with greater percent reduction in BP complexity between altitudes had greater percent reduction in $\mathrm{REM}$ ratio and $\mathrm{SpO}_{2}(\mathrm{r}=0.38 \sim 0.45, p=0.0090 \sim 0.0200)$, after adjustment for age, BMI, baseline apnea and altitude.

Conclusion: These results suggested that the characterization of BP complexity may provide novel insights into the underlying mechanisms through which the exposure to hypoxia affects cardiovascular health during sleep, as well as sleep quality. This BP complexity may serve as a novel marker to help the management of cardiovascular health and sleep quality in high-altitude living.

Keywords: high altitude, beat-to-beat blood pressure, multiscale entropy, sleep quality

\section{Introduction}

Good sleep quality is important for maintaining normal regulation of numerous essential physiological procedures, including the cardiovascular system. The exposure to hypoxia in a high-altitude environment has been evidenced to largely affect sleep quality, to induce severe sleep disturbances, such as insomnia, to be 
associated with problems in the cardiovascular system, and to worsen the symptoms in some diseases, such as insulin resistance in type-II diabetes mellitus. ${ }^{1-5}$ Studies have shown that, for example, compared to sleep in the plain level, people who are naïve to high-altitude environment often experience sleep problems, including less slow-wave sleep (SWS), more sleep disturbances and higher apnea hypopnea index (AHI) during sleeping at an altitude of $3500 \mathrm{~m} .{ }^{6}$ The exposure to hypoxia has also been linked to the altered regulation of the cardiovascular system, such as elevated blood pressure (BP) level in the daytime and the altered control of sympathetic nervous system. However, the effects of hypoxia on the characteristics of BP regulation during sleep, which are closely related to sleep quality, ${ }^{7}$ are not fully understood.

Traditionally, the characteristics of BP were measured based upon one single scale (eg, mean level and variability). ${ }^{8,9}$ However, BP is regulated continuously by a host of underlying physiologic procedures (eg, the neural impulse and secretion of neurochemical transmitters). These procedures interact with each other over multiple scales of time and space. The multiscale dynamics of BP regulation are thus "complex," containing patterns of physiologically meaningful patterns that are self-similar over multiple temporal scales. ${ }^{10-18}$ The degree of such physiologic complexity, which cannot be appropriately captured by those traditional metrics on one single scale, can be quantified using methods derived from complex systems theory. Multiscale entropy (MSE), for example, quantifies the recurrence of patterns, or entropy, in physiologic series at different scales of time or space. ${ }^{16,17}$ Lower complexity of the dynamics in the system, as quantified by MSE reflects poorer adaptability of the system to stressors (eg, alteration from internal and external factors) and functionality. Recently, studies have emerged to characterize the complexity of the continuous BP fluctuations at resting state, and have linked BP complexity to several important functionalities and the risk of neurodegenerative disorders (eg, dementia). ${ }^{12,14,15}$ For example, in a cohort of older adults, Jiang et al, observed that older adults with hypertension had lower BP complexity compared to normotensives, and lower BP complexity was associated with slower walking speed and elevated white matter lesions. ${ }^{14,15}$ These findings suggested that the characterization of BP complexity may provide novel insights into the pathological changes in cardiovascular system and helps the management of diseases. Still, the influence of exposing to hypoxic environment on the complexity of BP fluctuations during sleep, and the relationship between BP complexity and the sleep characteristics, eg, ratio of rapideye-movement (REM) sleep stage, are unknown.

In this study, we measured the characteristics of sleep, the oxygen saturation, and the continuous BP series during sleeping in the conditions of different altitude levels (ie, at 0 - (plain level), 2000-, and 4000-m conditions) in a group of younger adults. We then used MSE to quantify the complexity of the continuous beat-to-beat systolic (SBP) and diastolic (DBP) blood pressure. Our primary hypothesis is that compared to plain level (ie, $0 \mathrm{~m}$ ), the sleep quality, BP complexity, and oxygen saturation would decrease in high-altitude conditions. Secondarily, as the participants in the group had sleep apnea, we also explored the potential effects of sleep apnea on BP complexity, and if the hypoxiainduced changes in BP complexity would be associated with the changes in sleep quality and oxygen saturation.

\section{Methods}

\section{Participants}

The sample size of participants was determined by the results of one of our pilot studies showing that the difference in BP complexity was $7 \%$ and the conservative assumption that the standard deviation of within-subject change in BP complexity would be as high as $5 \%$. We estimated that complete datasets on 25 participants, accounting for $20 \%$ attrition, would provide $90 \%$ power to detect a mean difference of $7 \%$ between conditions with 0.05 type I error rate.

The inclusion criteria of the participant recruitment were: (1) of age between 18 to 30 years; (2) "naïve to high-altitude sleep", that is, living on the plain for the lifetime without previous experience of living in highaltitude areas; (3) willing and committed to complete all the study procedures. Those who met any of the following criteria were excluded: (1) any overt neurological diseases (eg, Parkinson's disease, stroke, or dementia); (2) severe sleep problems as assessed by the score of Pittsburgh Sleep Quality Index (PSQI) greater than 15; (3) hospitalized in the past three months; and (4) cannot understand the study protocol. A total of 25 younger men (age: 19 27 years) were eligible to be enrolled in this study.

\section{Ethics}

All experimental methods and protocols were approved by the Sports Science Experimental Institutional Review Board (IRB) of Beijing Sport University and carried out in 
accordance with relevant guidelines (No. 2021052H). All participants were informed about the study purpose and all the important information regarding safety. Then they provided written consent in order to participate in this study. None of the participants was aged under 18 years old.

\section{Study Protocol}

Each participant completed three study visits, each consisting of one nocturnal sleep, under three different altitudes of 0 (ie, plain), 2000, and $4000 \mathrm{~m}$, respectively. The sleep and BP assessments were completed in the hypoxic room of the Hypoxic Training Laboratory at Beijing Sport University. The room was designed to enable the adjustment of oxygenation level in the air by using the centralized oxygen filtration system (Low Oxygen Systems $\mathrm{GmbH}$, Berlin, Germany), thus simulating the hypoxic environment at different altitudes. All the participants completed the visits following the same order, that is, the first night was at altitude of 0 (ie, the oxygen concentration was set at $20.9 \%$ ), then at $2000 \mathrm{~m}$ (ie, the oxygen concentration was set at $16.3 \%$ ), and the third night at $4000 \mathrm{~m}$ altitude (ie, the oxygen concentration was set at $12.7 \%$ ). To try to eliminate the potential carry-over effects from the prior exposure to hypoxia condition, the first and second nights were separated by one week, and the second and third nights were separated by two weeks following the design of previous studies. ${ }^{17}$

\section{Experimental Procedure of Sleep Test}

On the day of each visit, participants were asked to keep regular diet of meals, but not to take coffee and alcohol within $12 \mathrm{~h}$ prior to the sleep test. They entered the study room at 10:00 pm of the day and were then instrumented with a 12-channel polysomnography (PSG) system (SOMNOscreen ${ }^{\mathrm{TM}}$ plus PSG+, Randersacker, Germany). The assessment of sleep characteristics was completed by this system following the standard PSG procedure. The metrics of sleep quality, including the ratio of the time at each sleep stage (ie, Stage 1, Stage 2, and Stage 3, and REM) to the whole sleep duration and the beginning and end time of sleep, were obtained. The oxygen concentration in the room was set to that at 0, 2000-, and 4000-m altitudes at the beginning of each visit, respectively. The room temperature was maintained at $25^{\circ} \mathrm{C}$. Then each participant was asked to try to get into sleep before 12:00 am and was woken up at 8:00 am in the morning of next day. Therefore, the length of the sleep duration was ensured at least eight hours. During the sleep, the PSG system recorded EEG signals by the scalp electrodes, and measured the continuous BP fluctuation and oxygen saturation $\left(\mathrm{SpO}_{2}\right)$ by using a finger-clip sensor on the middle finger of the left hand.

\section{Data Analysis}

The continuous beat-to-beat systolic (SBP) and diastolic (DBP) blood pressure series during sleep were sampled at $100 \mathrm{~Hz}$. The Domino ${ }^{\mathrm{TM}}$ software package (Somnomedics, Wurzburg, Germany) derived the values of SBP and DBP of each beat. This finger BP measurement technique was validated against invasive brachial artery pressure. ${ }^{13}$ Large variation or motion artifacts were observed in the beginning and at the end of the sleep test. To ensure high data quality (ie, signal-to-noise ratio) and remove the potential weakening period before sleep and after wake-up, we excluded the sampled points of BP in the first and last hour from each original BP record based upon the time stamps provided by the PSG system. Some outliers in the BP series of which the value was greater or lower than mean $\pm 2 \times$ standard deviation (SD) of the series were then interpolated by the mean of the whole BP fluctuation. All of these preprocessed BP recordings were then used in the following analyses.

\section{Multiscale Entropy (MSE) on BP Series}

The complexity of preprocessed SBP and DBP series was then quantified using multiscale entropy (MSE). MSE measures the multiscale dynamics in the pattern of physiological series. In the procedure of MSE calculation, the series were first divided into nonoverlapping windows of length equaling a scale factor, $\tau$ (ie, the "coarse-graining" step), and then the sample entropies of each "coarsegrained" series were calculated. ${ }^{14}$ Here we used scales ranging from 1 to 40 data points. Thus, in the coarsegraining process, the series at Scale 1 was the raw series, that at Scale 2 was constructed by averaging every two nonoverlapped points, consisting of half the number of points in the raw series. Sample entropy is defined by the negative natural logarithm of the conditional probability that a time series, having repeated itself within a tolerance $r$ for $m$ points (pattern length), will also repeat itself for $m$ +1 points without self-matches. The sample entropy of each coarse-grained series in this study was computed by choosing $\mathrm{m}=2$ and $\mathrm{r}=15 \%$, which was suggested by previous studies. ${ }^{14}$ The length of the BP series (ie, about eight hours) met the standard practice for obtaining reliable estimates of sample entropy. Finally, the BP complexity 
was defined as the averaged entropy across 40 scales. Lower MSE reflects lower complexity.

Additionally, the mean BP level were also obtained and used in the following analyses.

\section{Sleep Characteristics}

The metrics associated with sleep quality, including Apnea Hypopnea Index (AHI), average $\mathrm{SpO}_{2}$, REM ratio, light sleep ratio, and deep sleep ratio, were assessed by the PSG system, and used in the following analysis.

\section{Statistical Analysis}

Statistical analyses were performed with JMP Pro 14 software (SAS Institute, Cary NC, USA). The significance level was set to $p<0.05$.

To examine our primary hypothesis, we used two-way ANOVA models to assess the effects of hypoxia on complexity of SBP and DBP during sleep. The dependent variable was the SBP and DBP complexity in separate models. The model factor was the altitude (ie, 0, 2000, and $4000 \mathrm{~m}$ ). Since age and BMI may affect the BP regulation, we included them into the models as covariates. The effects of hypoxia on mean BP level, sleep characteristics (ie, AHI, ratio of REM, light, and deep sleep), and $\mathrm{SpO}_{2}$ were also examined using similar models.

Secondarily, the sleep apnea severity at baseline (ie, as measured at $0-\mathrm{m}$ condition) may potentially influence the effects of hypoxic environment on BP complexity and sleep characteristics, we thus categorized the severity of baseline sleep apnea into "normal" (AHI <5), "moderate (5-30)" and "severe (>30)" and used this categorical factor in the following models. Specifically, two-by-two ANOVA models were used to explore the contribution of sleep apnea to the effects of hypoxia on BP complexity. The dependent variable of each model was the SBP and DBP complexity. The model factors were altitude, baseline sleep apnea (ie, normal, moderate, and severe), and their interaction. Age and BMI were also included as covariates in these ANOVA models. Tukey's post hoc analysis was used to examine the location where the significance was shown between conditions and groups. Similar models were also used to examine the contribution of sleep apnea to the effects of hypoxia on sleep characteristics and $\mathrm{SpO}_{2}$.

Next, to explore the relationship between BP complexity and sleep characteristics, we used linear regression models to test (1) if at baseline (ie, 0 -m condition), the complexity of SBP and DBP were correlated with those sleep-related metrics at baseline; and (2) if the percent changes in BP complexity were correlated with the percent changes of the sleep characteristics from baseline to 2000- and 4000-m conditions. Similar models were used to examine the relationships between mean BP level and sleep-related metrics. All the regression models were adjusted for age, BMI, and baseline sleep apnea severity.

\section{Results}

All the participants (age (mean \pm standard deviation): $23.1 \pm 2.5$ years) successfully completed the study visits without reporting adverse events. Table 1 showed the demographics, sleep characteristics and BP metrics of the participants as measured at different conditions. At baseline (ie, 0 -m condition), eight participants were categorized as normal, nine were with moderate sleep apnea, eight were with severe sleep apnea. Between these three groups, no significant difference was observed in age, BMI, and baseline sleep characteristics $(p>0.30)$.

\section{The Effects of Different Altitudes and the Severity of Sleep Apnea on on BP Complexity}

The ANOVA models of the primary analysis showed that compared to $0-\mathrm{m}$ condition, both $\mathrm{SBP}(\mathrm{F}=11.4, p=0.0003)$ and DBP $(\mathrm{F}=12.1, p=0.0002)$ complexity were decreased at 2000-m and such decrease elevated significantly at 4000-m conditions, after adjusted for age and BMI. No such significant effects of hypoxia on mean BP level were observed $(\mathrm{F}<1.2, p>0.3200)$.

Similar results were observed in our secondary analysis. The two-by-two ANOVA models demonstrated the main effects of altitude and baseline sleep apnea severity on SBP and DBP complexity. Specifically, compared to $0-\mathrm{m}$ condition, the complexity of both SBP ( $\mathrm{F}=8.2$, $p=0.0009)$ and DBP $(\mathrm{F}=5.6, p=0.0007)$ significantly decreased at 2000-m and 4000-m conditions (Table 1). Significant effects of baseline apnea severity on SBP and DBP complexity were also observed, that is, across three altitude conditions, participants with more severity of sleep apnea at baseline had lower complexity at high-altitude conditions $(\mathrm{F}>5.5, p<0.0060)$. Tukey's post hoc analyses revealed that compared to 0 -m condition, lower BP complexity and lowest BP complexity were observed at 2000-m and 4000-m conditions, respectively; and compared to normal, those with moderate sleep apnea had lower BP complexity and those with severe apnea had the lowest BP complexity. No significant interaction between the conditions and apnea severity was observed. All these observed significant differences were independent from age, and BMI. 
Table I The Demographics, Sleep Characteristics and BP Metrics in Participants

\begin{tabular}{|c|c|c|c|c|c|}
\hline & & & \multicolumn{3}{|c|}{ Sleep Apnea Level } \\
\hline & & $\begin{array}{l}\text { Total } \\
(n=25)\end{array}$ & $\begin{array}{c}\text { Normal } \\
(n=8)\end{array}$ & $\begin{array}{c}\text { Moderate } \\
(n=9)\end{array}$ & $\begin{array}{l}\text { Severe } \\
(n=8)\end{array}$ \\
\hline \multicolumn{2}{|l|}{ Age (years) } & $23.1 \pm 2.5$ & $24.4 \pm 1.9$ & $23.2 \pm 2.5$ & $21.8 \pm 2.5$ \\
\hline \multicolumn{2}{|l|}{ BMI } & $23.0 \pm 1.7$ & $23.6 \pm 1.4$ & $22.4 \pm 1.7$ & $23.1 \pm 1.7$ \\
\hline \multirow[t]{3}{*}{$\mathrm{AHI}$} & 0 & $20.3 \pm 27.1^{\mathrm{a}}$ & $2.5 \pm 1.1$ & $9.9 \pm 3.5$ & $47.5 \pm 33.1$ \\
\hline & 2000 & $30.4 \pm 17.1^{\mathrm{b}}$ & $28.2 \pm 19.8$ & $29.6 \pm 13.4$ & $33.6 \pm 18.6$ \\
\hline & 4000 & $39.6 \pm 17.4^{c}$ & $43.4 \pm 13.2$ & $43.6 \pm 23.5$ & $31.3 \pm 10.9$ \\
\hline \multirow[t]{3}{*}{ Light sleep ratio (\%) } & 0 & $44.6 \pm 11.4$ & $43.5 \pm 13.8$ & $43.6 \pm 9.6$ & $46.8 \pm 11.7$ \\
\hline & 2000 & $42.4 \pm \mid 2.1$ & $43.3 \pm 8.0$ & $37.8 \pm 15.1$ & $46.8 \pm 11.3$ \\
\hline & 4000 & $42.1 \pm 11.8$ & $47.0 \pm 11.9$ & $35.6 \pm 10.3$ & $44.6 \pm 11.3$ \\
\hline \multirow{3}{*}{$\begin{array}{l}\text { Deep sleep ratio } \\
\text { (\%) }\end{array}$} & 0 & $24.0 \pm 12.8$ & $28.4 \pm 11.7$ & $13.4 \pm 8.9$ & $31.4 \pm 10.4$ \\
\hline & 2000 & $27.8 \pm 9.4$ & $31.1 \pm 6.8$ & $20.2 \pm 8.2$ & $32.9 \pm 8.1$ \\
\hline & 4000 & $29.6 \pm 10.4$ & $30.4 \pm 11.4$ & $25.8 \pm 9.9$ & $33.1 \pm 9.9$ \\
\hline \multirow[t]{3}{*}{ REM ratio } & 0 & $10.2 \pm 5.8^{\mathrm{a}}$ & $8.1 \pm 4.0$ & $14 \pm 5.8$ & $7.9 \pm 5.7$ \\
\hline & 2000 & $8.0 \pm 4.9^{b}$ & $7.8 \pm 4.1$ & $9.9 \pm 4.5$ & $6.3 \pm 5.8$ \\
\hline & 4000 & $6.2 \pm 4.4$ & $5.0 \pm 4.0$ & $8.2 \pm 5.2$ & $5.1 \pm 3.1$ \\
\hline \multirow[t]{3}{*}{$\mathrm{SpO}_{2}$} & 0 & $96.5 \pm 1.0^{\mathrm{a}}$ & $96.4 \pm 0.9$ & $96.6 \pm 1.5$ & $96.6 \pm 0.4$ \\
\hline & 2000 & $91.6 \pm 2 .^{b}$ & $90.3 \pm 3.4$ & $92.7 \pm 1.5$ & $91.9 \pm 2.0$ \\
\hline & 4000 & $86.8 \pm 4.9^{c}$ & $84.4 \pm 4.3$ & $86.8 \pm 3.3$ & $89.3 \pm 6.1$ \\
\hline \multirow[t]{3}{*}{ Mean BP level } & 0 & $77.4 \pm 10.5$ & $73.8 \pm 12.6$ & $80.0 \pm 9.8$ & $78.1 \pm 9.1$ \\
\hline & 2000 & $70.5 \pm 10.6$ & $71.0 \pm 9.2$ & $70.3 \pm 9.7$ & $70.3 \pm 13.9$ \\
\hline & 4000 & $75.7 \pm 9.5$ & $74.4 \pm 9.6$ & $73.0 \pm 11.5$ & $79.9 \pm 6.3$ \\
\hline \multirow[t]{3}{*}{ SBP complexity } & 0 & $1.28 \pm 0.28^{\mathrm{a}}$ & $|.32 \pm 0.3|$ & $1.29 \pm 0.25$ & $1.25 \pm 0.33$ \\
\hline & 2000 & $1.20 \pm 0.38^{\mathrm{b}}$ & $1.28 \pm 0.37$ & $1.19 \pm 0.29$ & $1.09 \pm 0.36$ \\
\hline & 4000 & $1.12 \pm 0.35^{c}$ & I. $18 \pm 0.44$ & $|| I \pm 0.3 \mid$. & $1.09 \pm 0.27$ \\
\hline \multirow[t]{3}{*}{ DBP complexity } & 0 & $1.32 \pm 0.26^{\mathrm{a}}$ & $1.34 \pm 0.33$ & $1.31 \pm 0.23$ & $|.28 \pm 0.3|$ \\
\hline & 2000 & $1.24 \pm 0.34^{\mathrm{b}}$ & $1.27 \pm 0.29$ & $1.22 \pm 0.33$ & $1.19 \pm 0.46$ \\
\hline & 4000 & $1.13 \pm 0.32^{c}$ & $1.19 \pm 0.36$ & $1.13 \pm 0.26$ & $1.11 \pm 0.29$ \\
\hline
\end{tabular}

Note: Different superscript letters (ie, a, b, and c) showed the significant difference $(p<0.05)$ between conditions. Specificially, the a and b in REM ratio reflected $p<0.0 \mathrm{I}$; and the a, b, and $c$ in AHI, SpO2, SBP and DBP complexity reflected $p<0.00 \mathrm{I}$.

Abbreviations: $\mathrm{AHI}$, hypoxia apnea index; $\mathrm{SpO}_{2}$, oxygen saturation; REM, rapid eye movement; SBP, systolic blood pressure; DBP, diastolic blood pressure.

\section{The Effects of Different Altitudes and the Severity of Sleep Apnea on Sleep Characteristics}

The models of primary analyses showed significant effects of altitude on sleep apnea, $\mathrm{SpO}_{2}$ and the REM ratio. Specifically, compared to baseline (ie, 0-m altitude), the AHI was higher at $2000-\mathrm{m}$, and highest at $4000-\mathrm{m}$ condition $(\mathrm{F}=8.8, p=0.0004)$; the $\mathrm{SpO}_{2}(\mathrm{~F}=72.1, p<0.0001)$ and REM ratio $(\mathrm{F}=64.2, p<0.0090)$ were decreased at $2000-\mathrm{m}$ and 4000-m conditions. No effects of altitude on light and deep sleep ratio were observed $(\mathrm{F}<2.3, p>0.2400)$.
The results of the secondary analysis also demonstrated the main effects of altitude on $\mathrm{SpO}_{2}(\mathrm{~F}=69.1, p<0.0001)$, and REM ratio $(\mathrm{F}=3.7, p=0.0300)$; and the main effects of the baseline sleep apnea severity on $\mathrm{SpO}_{2}(\mathrm{~F}=4.4, p=0.0100)$, light sleep $(\mathrm{F}=4.8, p=0.0100)$, deep sleep $(\mathrm{F}=9.2, p=0.0004)$ and $\mathrm{REM}$ ratio $(\mathrm{F}=3.6, p=0.0300)$ (Table 1$)$. Post hoc analyses revealed elevated changes in these metrics (Table 1). Specifically, compared to $0-\mathrm{m}$ condition, the $\mathrm{SpO}_{2}$ was lower at 2000-m condition and lowest at 4000-m condition ( $\mathrm{F}=55.1, p<0.0001$ ); the ratio of REM period decreased from $10 \%$ to $8 \%$ at $2000-\mathrm{m}$, and further decreased to $6 \%$ at 4000-m condition $(\mathrm{F}=5.0, p=0.0090$, Figure 1); compared 

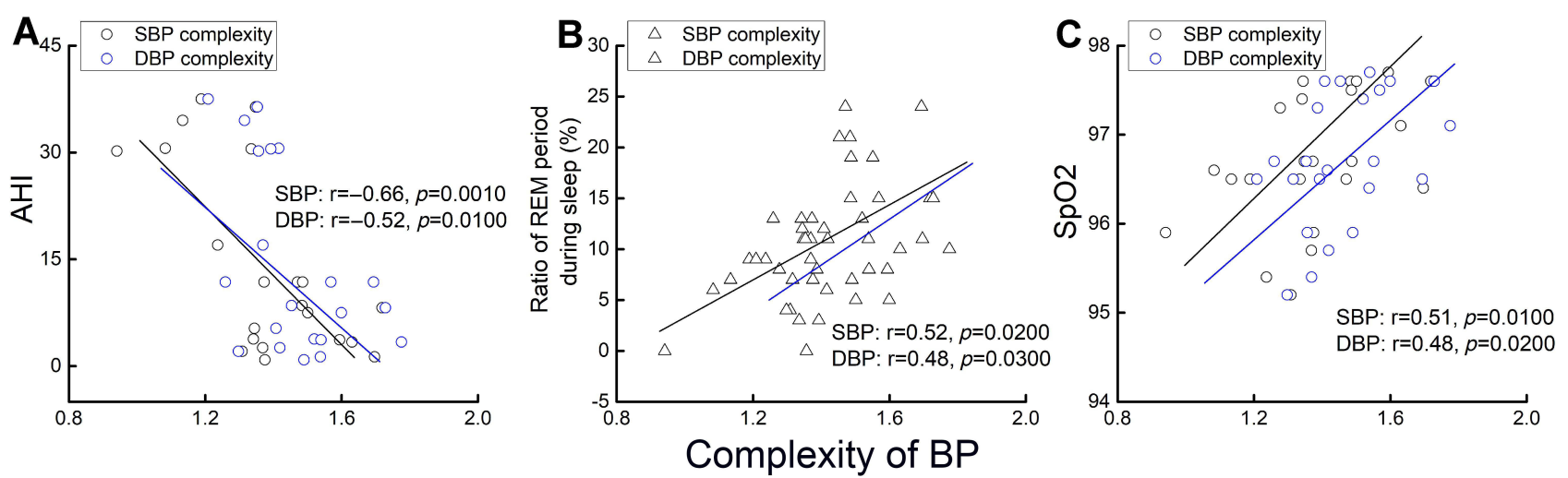

Figure I The association between the complexity of $\mathrm{BP}$ and $\mathrm{AHI}, \mathrm{REM}$ ratio and $\mathrm{SpO}_{2}$ at baseline. Across all the participants, those with lower complexity of SBP and/or DBP had greater AHI (ie, greater severity of sleep apnea) (A), lower ratio of REM (B) and lower $\mathrm{SpO}_{2}$ level (C).

to normal, lowest level of $\mathrm{SpO}_{2}$, REM ratio, light sleep ratio, and deep sleep ratio, and lower levels of these metrics were observed in participants with moderate, and with severe sleep apnea, respectively. A trend towards significance in the effects of altitude on deep sleep ratio was also observed $(\mathrm{F}=2.6, p=0.0800)$. No significant effect of the altitude on the light sleep ratio was shown $(\mathrm{F}=0.5, p=0.6400)$, and no significant interaction between altitude and sleep apnea on these outcomes was observed $(\mathrm{F}<1.8, p>0.1100)$

\section{The Relationship Between BP Complexity and Sleep Characteristics}

The linear regression analysis adjusted for age, BMI, altitude, and baseline apnea severity showed that at baseline, the complexity of SBP and DBP was significantly correlated with $\mathrm{AHI}, \mathrm{SpO}_{2}$ and the ratio of REM (Figure 1). Specifically, participants with lower complexity of SBP and/or DBP had greater AHI (SBP: $\mathrm{r}=-0.66, p=0.0010$; DBP: $\mathrm{r}=-0.52, p=0.0100$ ), lower ratio of REM (SBP: $\mathrm{r}=0.52, p=0.0200 ; \mathrm{DPB}: \mathrm{r}=0.48, p=0.0200)$ and lower $\mathrm{SpO}_{2}$ (SBP: $\mathrm{r}=0.51, p=0.0100$; DBP: $\mathrm{r}=0.48, p=0.0200$ ).

In addition, those with a greater percent reduction in BP complexity from baseline to $2000-\mathrm{m}$ and/or $4000-\mathrm{m}$ conditions also had a greater percent reduction in REM ratio and $\mathrm{SpO}_{2}$ level $(\mathrm{r}=0.38 \sim 0.45, p=0.0090 \sim 0.0200$, Figure 2), and this association was independent from age, BMI, baseline apnea and altitude. No significant association was observed between the percent change of BP complexity and that in AHI $(r<0.22, p>0.5100)$.

\section{Discussion}

In this pilot study, we quantified the multiscale dynamics of continuous BP fluctuations in a group of younger adults with normal to severe sleep apnea in different hypoxic conditions. The results showed that (1) the elevated hypoxia decreased the SBP and DBP complexity during sleep and impaired the sleep characteristics (eg, increased AHI), (2) the baseline sleep apnea may also alter the SBP and DBP complexity, and (3) BP complexity and its changes were associated with important sleep characteristics (ie, $\mathrm{SpO}_{2}, \mathrm{REM}$ ratio, $\mathrm{AHI}$ ) and their changes in hypoxic conditions, respectively. These findings revealed that the BP complexity may capture the physiological changes in the cardiovascular system induced by hypoxia, which are important to the regulation of sleep, and may provide novel and unique insights into the underlying mechanisms through which the exposure to hypoxia affects sleep quality. This BP complexity metric holds great promise to help optimize the management of cardiovascular health and sleep when facing the challenges of hypoxic exposure.

We here observed the effects of hypoxia on BP regulation as characterized by BP complexity, that is, the BP complexity is decreased when people suffering from exogenous (high altitude) and/or endogenous (sleep apnea) hypoxia. The theory of physiologic complexity has linked the degree of the complexity of the spontaneous fluctuation in a given physiologic system to the capacity of this system to adapt to both external and internal stressors. The effects of both exogenous (ie, hypoxia due to high altitudes) and endogenous (ie, sleep apnea) types of the stressors on BP complexity are examined in this study. We provide novel proof of its concept that the exposure to hypoxia significantly diminishes BP complexity during sleep, and the changes in BP complexity are associated with the changes in sleep characteristics. Conversely, no 
A

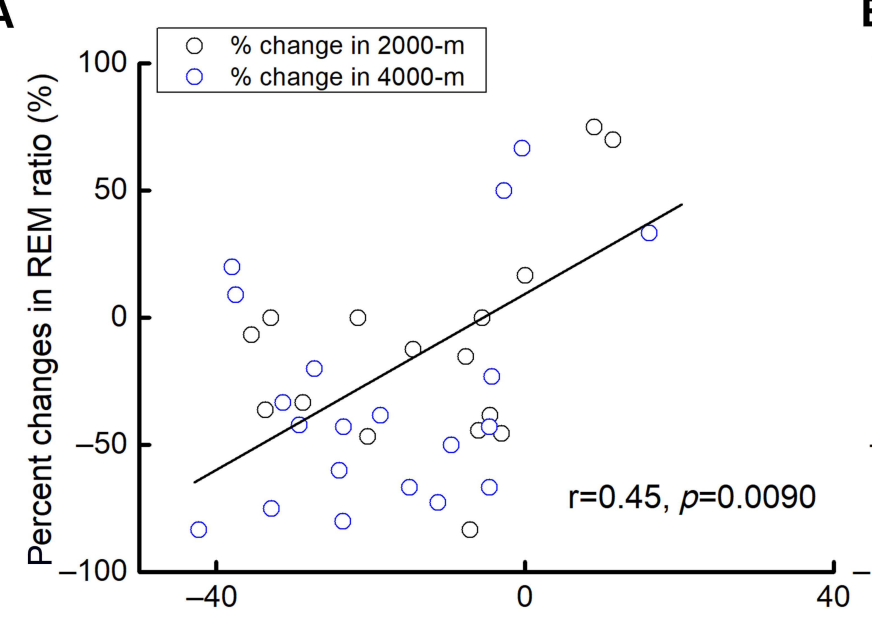

C

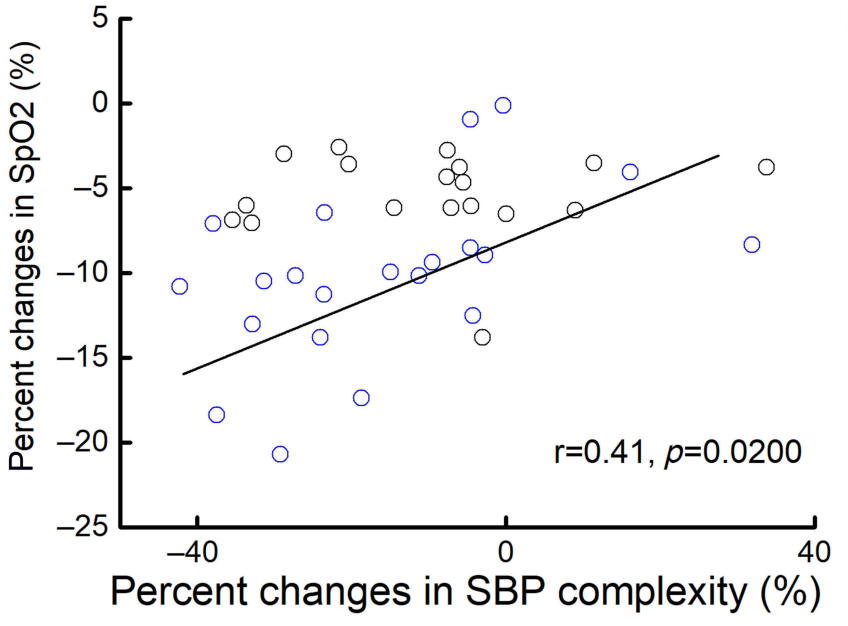

B

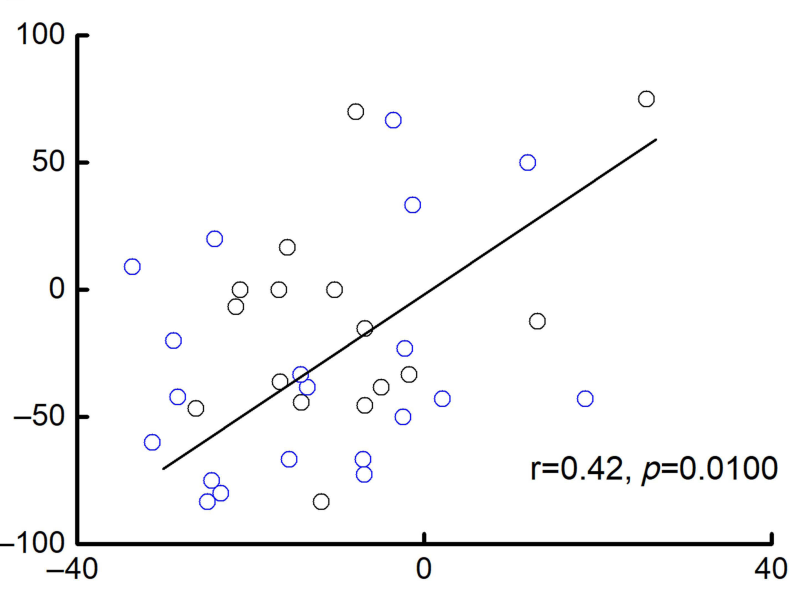

Figure 2 The association between the percent changes in SBP and DBP complexity and the percent changes in REM ratio (A and $\mathbf{B})$ and SpO 2 (C and $\mathbf{D})$ due to the

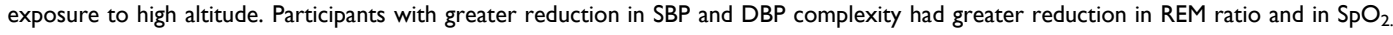

such significant relationship between traditional measures of BP (eg, mean BP level) and sleep characteristics is observed. These observations reveal that the interference of these two types of stressors on the regulation of the cardiovascular system pertaining to sleep physiology happens over multiple temporal scales, and the multiscale dynamics of BP, as quantified by BP complexity, can capture such important functional and physiological inference, while traditional measures based upon one single scale cannot. It also suggests that this BP complexity metric, as derived from a relatively short-term BP measurement (ie, in several minutes in previous studies ${ }^{12,14,15}$ or hours in this study), can help assess the subtle physiological and pathologic changes in cardiovascular system and help optimize the management of cardiovascular health and the related functionalities (eg, walking speed $^{14}$ ) in future's clinics. It is interesting that in our secondary analysis, no interactions between the high altitude and sleep apnea on the BP complexity was observed, which may be due to the small sample size in this pilot study.

The oxygen supply and saturation are important for maintaining fundamental activities of human beings, including the regulation of sleep. The oxygen supply depends upon the regulation of blood flow, including blood pressure, in vascular systems. The BP regulation is maintained by numerous neurophysiological components that are interacting and communicating with each other over multiple scales of time and space. ${ }^{13}$ The multiscale dynamics of BP fluctuation, as assessed by a complexity metric (such as the MSE we used here), may thus contain critical information pertaining to those multiscale control mechanisms. Herein for the first time, we characterize the complexity of continuous BP fluctuation during sleep and explored the association between BP complexity and the level of oxygen saturation assessed by level of $\mathrm{SpO}_{2}$. It is 
observed that at baseline, BP complexity is associated with $\mathrm{SpO}_{2}$; and their changes due to hypoxia were also associated with each other, revealing that the multiscale dynamics of BP fluctuation, as measured by complexity of continuous BP, contribute significantly to one's capacity of oxygen saturation during sleep.

The pathophysiology of obstructive sleep apnea (OSA) is multifactorial, including several physiological "traits", 19,20 such as poor upper airway anatomy that predisposes the airway, and diminished ability of the muscles to respond to a respiratory challenge (eg, reduced oxygen in the highaltitude conditions). Low threshold of arousal which may cause the person to arouse from sleep, and a hypersensitive ventilatory control system are often referred to as a system with a high loop gain (LG). Multiple studies have shown the interference of hypoxia on sleep quality. In a recent study, Edwards et al, for example, demonstrated that in middle-aged people (age $>50$ years) with OSA, the exposure to hypoxia may worsen the sleep apnea by increasing LG and the arousal threshold in addition to improving pharyngeal collapsibility. ${ }^{21}$ Here we provided confirmatory and complementary evidence that in a group of younger adults with and without sleep apnea, the exposure to exogenous hypoxia (ie, high altitude) elevated the grade of AHI; and both exogenous and endogenous hypoxia significantly alter the sleep characteristics, including reduced deep sleep and REM stage ratio.

These results also suggest that rehabilitative strategies for cardiovascular health in those with sleep apnea may be optimized by targeting the multiscale dynamics of BP fluctuation. Recent studies have shown that the loss of physiologic complexity is not obligatory but can be restored with appropriate interventions. ${ }^{18,22,23}$ Zhou et al, for example, showed that using sub-threshold vibratory stimulation on foot soles may enhance the foot-sole tactile function and thus improve the complexity of standing postural control. ${ }^{23}$ Future studies are thus worthwhile to explore the effects of interventions (eg, continuous positive airway pressure) on the complexity of BP fluctuation during sleep, and to examine if an increase in the BP complexity during sleep is associated with improvements in sleep quality in patients with sleep apnea.

Several limitations should be noted in this pilot study. Only 25 younger men were recruited. The gender bias should also be noted. Therefore, future studies with a large sample size and matched number of men and women are needed to confirm the findings in this study. The characteristics of the cerebral blood flow during sleep are also needed to be measured in future studies to help us better understand the potential pathway through which the abnormality in the cardiovascular system affects the cerebral function in those suffering from sleep apnea and those exposed to high-altitude hypoxia.

Nevertheless, this pilot study provides a novel measure of the BP regulation (ie, BP complexity) during sleep and the preliminary evidence of the effects of hypoxia on BP regulation, and of the association between BP complexity and oxygen saturation and sleep characteristics. These observations indicate that BP complexity may be a novel marker that can help us understand and manage the influence of hypoxia on sleep.

\section{Acknowledgment}

Zhenxiang Guo is now affiliated with the Department of Physical Education, Nanjing University of Aeronautics and Astronautics, Nanjing, JiangSu, China, 210016.

\section{Funding}

This study was supported by Specialized Research Fund for the Doctoral Program of Higher Education of China (20131112120007).

\section{Disclosure}

The authors report no conflicts of interest in this work.

\section{References}

1. Ainslie PN, Lucas SJ, Burgess KR. Breathing and sleep at high altitude. Respir Physiol Neurobiol. 2013;188(3):233-256. doi:10.1016/j.resp.2013.05.020

2. Bloch KE, Buenzli JC, Latshang TD, Ulrich S. Sleep at high altitude: guesses and facts. $J$ Appl Physiol. 2015;119(12):1466-1480. doi:10.1152/japplphysiol.00448.2015

3. Kimura H, Ota H, Kimura Y, Takasawa S. Effects of intermittent hypoxia on pulmonary vascular and systemic diseases. Int $J$ Environ Res Public Health. 2019;16:17. doi:10.3390/ijerph16173101

4. Gabryelska A, Karuga FF, Szmyd B, Bialasiewicz P. HIF-1alpha as a mediator of insulin resistance, T2DM, and its complications: potential links with obstructive sleep apnea. Front Physiol. 2020;11:1035. doi:10.3389/fphys.2020.01035

5. Szmyd B, Rogut M, Bialasiewicz P, Gabryelska A. The impact of glucocorticoids and statins on sleep quality. Sleep Med Rev. 2021;55:101380. doi:10.1016/j.smrv.2020.101380

6. Johnson PL, Edwards N, Burgess KR, Sullivan CE. Sleep architecture changes during a trek from 1400 to $5000 \mathrm{~m}$ in the Nepal Himalaya. $J$ Sleep Res. 2010;19(1 Pt 2):148-156. doi:10.1111/j.13652869.2009.00745.x

7. Tremblay JC, Boulet LM, Tymko MM, Foster GE. Intermittent hypoxia and arterial blood pressure control in humans: role of the peripheral vasculature and carotid baroreflex. Am J Physiol Heart Circ Physiol. 2016;311(3):H699-706. doi:10.1152/ajpheart.00388.2016 
8. Horiuchi M, Oda S, Uno T, Endo J, Handa Y, Fukuoka Y. Effects of short-term acclimatization at the summit of Mt. Fuji $(3776 \mathrm{~m})$ on sleep efficacy, cardiovascular responses, and ventilatory responses. High Alt Med Biol. 2017;18(2):171-178. doi:10.1089/ham.2016.0162

9. Sasaki N, Nagai M, Mizuno H, Kuwabara M, Hoshide S, Kario K. Associations between characteristics of obstructive sleep apnea and nocturnal blood pressure surge. Hypertension. 2018;72 (5):1133-1140. doi:10.1161/HYPERTENSIONAHA.118.11794

10. Weber MA. Blood pressure variability and cardiovascular prognosis: implications for clinical practice. Eur Heart J. 2017;38 (37):2823-2826. doi:10.1093/eurheartj/ehx322

11. Magder S. The meaning of blood pressure. Crit Care. 2018;22 (1):257. doi:10.1186/s13054-018-2171-1

12. Ma Y, Zhou J, Kavousi M, et al. Decreased complexity and increased variability in systolic blood pressure are associated with elevated long-term risk of dementia: the Rotterdam Study: epidemiology: cardiovascular risk factors. Alzheimer's \& Dementia. 2020;16: e041587. doi:10.1002/alz.041587

13. Manuel J, Färber N, Gerlach DA, et al. Deciphering the neural signature of human cardiovascular regulation.eLife. Sciences. 2020;9:e55316

14. Jiang X, Cai Y, Zhao Y, et al. The complexity of blood pressure fluctuation mediated the effects of hypertension on walking speed in older adults. Front Aging Neurosci. 2021. doi:10.3389/ fnagi.2021.640942

15. Jiang X, Guo Y, Zhao Y, et al. Multiscale dynamics of blood pressure fluctuation is associated with white matter lesion burden in older adults with and without hypertension: observations from a pilot study. Front Cardiovasc Med. 2021;(8):80.
16. Busa MA, van Emmerik REA. Multiscale entropy: a tool for understanding the complexity of postural control. J Sport Health Sci. 2016;5(1):44-51. doi:10.1016/j.jshs.2016.01.018

17. Bao D, Chen Y, Yue H, Zhang J, Hu Y, Zhou J. The relationship between multiscale dynamics in tremulous motion of upper limb when aiming and aiming performance in different physical load conditions. J Sports Sci. 2019;37(22):2625-2630. doi:10.1080/ 02640414.2019 .1651591

18. Zhou J, V P, Wooten T, et al. Multiscale dynamics of spontaneous brain activity is associated with walking speed in older adults. $J$ Gerontol a Biol Sci Med Sci. 2020;75(8):1566-1571.

19. Bradley TD, Floras JS. Obstructive sleep apnoea and its cardiovascular consequences. Lancet. 2009;373(9657):82-93. doi:10.1016/ S0140-6736(08)61622-0

20. Lévy P, Kohler M, McNicholas WT, et al. Obstructive sleep apnoea syndrome. Nat Rev Dis Primers. 2015;1:15015.

21. Edwards BA, Sands SA, Owens RL, et al. Effects of hyperoxia and hypoxia on the physiological traits responsible for obstructive sleep apnoea. $J$ Physiol. 2014;592(20):4523-4535. doi:10.1113/ jphysiol.2014.277210

22. Zhou J, Lipsitz L, Habtemariam D, Manor B. Sub-sensory vibratory noise augments the physiologic complexity of postural control in older adults. $J$ Neuroeng Rehabil. 2016;13(1):44. doi:10.1186/ s12984-016-0152-7

23. Liu Z, Ma H, Poole V, et al. Effects of multi-session repetitive transcranial magnetic stimulation on motor control and spontaneous brain activity in multiple system atrophy: a Pilot Study. Front Behav Neurosci. 2018;12:90. doi:10.3389/fnbeh.2018.00090

\section{Publish your work in this journal}

Nature and Science of Sleep is an international, peer-reviewed, open access journal covering all aspects of sleep science and sleep medicine, including the neurophysiology and functions of sleep, the genetics of sleep, sleep and society, biological rhythms, dreaming, sleep disorders and therapy, and strategies to optimize healthy sleep.
The manuscript management system is completely online and includes a very quick and fair peer-review system, which is all easy to use. Visit http://www.dovepress.com/testimonials.php to read real quotes from published authors. 